\title{
Fidelidad de la
}

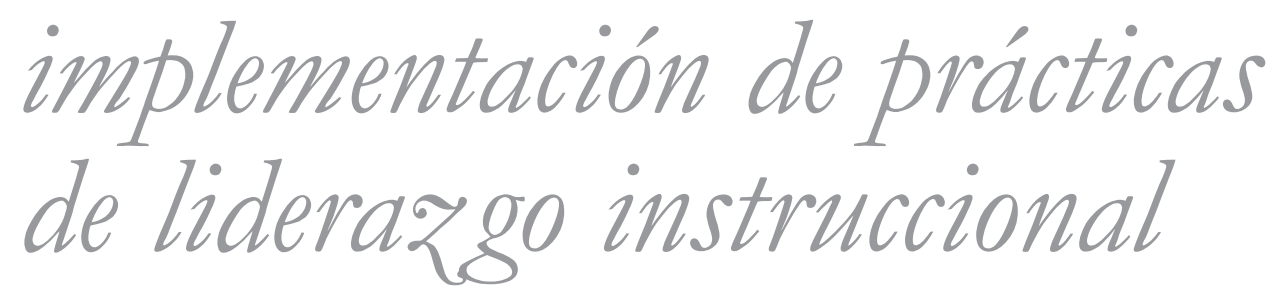

Fidelite de l'implementation des pratiques de leadership d'instruction

Fidelidade da implementação de práticas de liderança instrucional

Fecha de recepción: 20 DE FEBRERO DE 2013 / Fecha de aceptación: 10 DE NOVIEMBRE DE 2014

/ Fecha de disponibilidad en línea: 25 DE MARZO DE 2015

Encuentre este artículo en http://magisinvestigacioneducacion.javeriana.edu.co/

doi:10.11144/Javeriana.M7-15.FIPL

Escrito por GERMÁN RODOLFO FROMM-RIHM

Pontificia Universidad Católica de Chile

Macul, SAntiago de Chile

grfromm@uc.cl

Ingrid Marie Olbrich-Guzmán Pontificia Universidad Católica de Chile

Macul, Santiago de Chile iolbrich@uc.cl

Paulo Luis Volante-Beach Pontificia Universidad Católica de Chile

Macul, Santiago de Chile pvolante@uc.cl

\section{Resumen}

Este artículo de investigación describe un estudio de fidelidad de la implementación (FI) de un conjunto de prácticas de liderazgo instruccional propuestas a equipos de directivos y docentes de seis escuelas secundarias de la Región Metropolitana en Chile. La introducción precisa este objetivo y la investigación en la que se enmarca este reporte. La metodología explica el marco conceptual que fundamenta la construcción de los conceptos evaluados y el levantamiento y análisis de la información. Los resultados diferencian la fidelidad alcanzada en cada colegio, de mayor fidelidad estructural que procedimental, con fortalezas en prácticas de monitoreo.

\section{Palabras clave descriptor}

Fidelidad de la implementación (FI), liderazgo instruccional, equipos de liderazgo.

\section{Transferencia a la práctica}

Esta investigación repercute en los proyectos de intervención en educación, pues resalta que una herramienta como la fidelidad de la implementación (FI) facilita la validez interna y permite responder ante los resultados que se obtienen. En este sentido, ejemplifica cómo relacionar una intervención con resultados posteriores. Para la práctica investigativa hace un aporte doble, en cuanto a la aplicación de la teoría desarrollada en el campo y al proporcionar datos para orientar trabajos futuros. En términos de las políticas para proyectos de mejora educativa, se propone como referente para tomar decisiones mejor informadas y de mayor validez técnica.

Para citar este artículo / To cite this article / Pour citer cet article / Para citar este artigo

Fromm,G., Olbrich, I. \& Volante, P. (2015). Fidelidad de la implementación de prácticas de liderazgo instruccional. magis, Revista Internacional de Investigación en Educación, 7 (15), 117-134. 


\section{Key words plus}

Implementation Fidelity, Instructional

Leadership, Leadership Teams.

\section{Abstract}

This research paper describes a study of implementation fidelity (FI) of a set of instructional leadership practices proposed to principals and teachers of six second ary schools in the Metropolitan Region in Chile. The introduction aims this objective as well as the research on which this report is framed. The methodology explains the conceptual framework underlying the construction of the concepts evaluated the collection and analysis of information. The results differentiate fidelity achieved in each school, presenting greater structura fidelity procedures, with strengths in monitoring practices.

\section{Transfer to practice}

This research influences the educational intervention projects by highlighting that a tool like implementation fidelity facilitates internal validity and allows to respond to the results obtained. In this sense, it exemplifies how to relate an intervention with subsequent outcomes. For research practice, this paper contributes to the application of the theory developed in the field and provides data to guide future works. Regarding the policies for educational improvement projects it is a reference for making better informed and more technical validity decisions.

\section{Mots clés descripteur}

Fidélité de l'implémentation, leadership

d'instruction, équipes de leadership.

\section{Résumé}

Cet article de recherche décrit une étude de fidélité de l'implémentation ( $F I)$ d'un ensemble de pratiques de leadership d'instruction proposées à équipes de directifs et enseignants de six écoles secondaires de la Région Métropolitaine au Chili. L'introduction précise cet objectif et la recherche dans laquelle ce rapport est cadré. La méthodologie explique le cadre conceptuel qui est le fondement de la construction des concepts évalués, I'accueil et l'analyse des données. Les résultats présentés montrent la différence de la fidélité dans chaque collège, en montrant plus fidélité structurale que de procédure, avec les point forts dans les pratiques de monitorage.

\section{Transfert à la pratique}

Cette recherche a une répercussion dans les projets d'intervention en éducation car elle souligne qu'un outil comme la fidélité de l'implémentation facilité la validité interne et permet de répondre face aux résultats qu'on obtienne. Dans ce sens, elle illustre comment faire le rapport entre une intervention avec les résultats postérieures. Pour la pratique de la recherche elle est une contribution double en ce qui concerne l'application de la théorie développée dans le terrain et qui fournit les données pour orienter les travails futurs. Dans les termes des politiques pour les projets de progrès éducatifs on la propose en tant qu'un référant pour prendre de décisions mieux informées et de plus grande validité technique.

\section{Palavras-chave descritor}

Fidelidade da implementação, liderança instrucional, equipes de liderança.

\section{Resumo}

Este artigo de pesquisa descreve um estudo de fidelidade da implementação (FI) de um conjunto de práticas de liderança instrucional propostas a equipes de diretores docentes de 6 escolas secundárias da Região Metropolitana no Chile. A introdução precisa este objetivo e a pesquisa na qual se enquadra este relatório. A metodologia explica o marco conceitual que fundamenta a construção dos conceitos avaliados, o levantamento e a análise da informação. Os resultados apresentados diferenciam a fidelidade atingida em cada colégio, apresentando maior fidelidade estrutural que procedimental, com fortalezas em práticas de monitoramento.

\section{Transferência à prática}

Esta pesquisa tem sua repercussão nos projetos de intervenção em educação por realçar que uma ferramenta como a fidelidade da implementação facilita a validade interna e permite responder ante os resultados obtidos. Neste sentido exemplifica como relacionar uma intervenção com resultados posteriores. Para a prática investigativa terminar por oferecer uma dupla contribuição tanto quanto à aplicação da teoria desenvolvida no campo como em proporcionar dados para orientar trabalhos futuros. Nos termos das políticas para projetos de meIhora educativa propõe-se como um referente para tomar decisões melhor informadas e de maior validade técnica. 


\section{Introducción}

El presente artículo describe un estudio de fidelidad de la implementación (FI) de un conjunto de prácticas de liderazgo instruccional propuestas a directivos y docentes de seis establecimientos escolares secundarios de la Región Metropolitana en Chile, a partir de la investigación Influencia del liderazgo instruccional en motivación y logro académico en estudiantes secundarios (2010-2013), la cual fue financiada por Fondecyt Iniciación No. 11100466, a quien se agradece el apoyo. En el subestudio presentado en este artículo se propone un diseño para evaluar la fidelidad de la implementación del proyecto mayor, considerando antecedentes conceptuales recientes de la literatura de $\mathrm{Fl}$, especialmente relevantes para su aplicación en intervenciones en organizaciones educativas.

\section{Objetivos}

El propósito de este estudio de fidelidad de implementación fue diseñar, implementar y evaluar indicadores de la intervención propuesta en el proyecto Equipos de Liderazgo Instruccional, en los establecimientos escolares en que se aplica el modelo y prácticas de liderazgo instruccional para la mejora de los aprendizajes (Volante, 2011). Para ello, en este estudio se busca cumplir con los siguientes objetivos específicos:

- Reportar los niveles de Fl del proyecto Influencia del liderazgo instruccional en motivación y logro académico en estudiantes secundarios.

- Diferenciar los niveles de fidelidad de la implementación que se dan en los seis establecimientos participantes de la intervención.

\section{Antecedentes conceptuales y empíricos}

\section{Fidelidad de la implementación}

Para cumplir con el propósito de este trabajo fue necesario sistematizar antecedentes sobre fidelidad de la implementación (FI), línea de investigación con más de 35 años de desarrollo en los campos de la salud pública, la salud mental y la educación, en la cual específicamente se ha reportado mayor insuficiencia para dar cuenta de la amplia variedad de iniciativas existentes (Mowbray, Holter, Teague \& Bybee, 2003). Además, el trabajo en el campo de la educación se ha centrado más en programas de implementación curricular (O’Donnell, 2008), con lo que queda aún más desprovista el área de intervenciones organizacionales o culturales en las escuelas.

En general, se han evitado definiciones exactas de $\mathrm{Fl}$, aunque siempre se la interpreta como el grado de ajuste entre la intención original de un programa de intervención y su puesta en práctica. Century, Rudnick y Freeman (2010) proponen la siguiente definición en su metaanálisis: "el grado de consistencia entre un programa realizado y el modelo del programa intencionado". Cuando la FI se utiliza con fines de investigación -sobre todo cuando se miden efectos de los programas- se puede entender como la confirmación de que la variable independiente operó según lo planeado (Moncher \& Prinz, 1991).

En este sentido, considerando programas de intervención que se conceptualizan como una variable independiente para lograr resultados específicos, la Fl resuelve la necesidad de constatar la validez interna. En términos prácticos, la medición de la fidelidad permite responder ante resultados ambiguos o negativos que no coinciden con las expectativas del
Descripción del artículo | Article description | Description de l'article | Artigo descrição

Este artículo se deriva del proyecto Influencia del liderazgo instruccional en motivación y logro académico en estudiantes secundarios (Fondecyt Iniciación №11100466). Este proyecto fue financiado por el Fondo Nacional de Desarrollo Científico, Tecnología e Innovación Tecnológica (Fondecyt), a quienes se agradece por hacer posible la investigación. Este estudio cualitativo es parte de una investigación cuasiexperimental desarrollada entre 2011 y 2013, que buscó aumentar la influencia instruccional de estos equipos en la motivación y rendimiento en matemáticas de los estudiantes de la cohorte 2010-2013. Para ello se creó el diseño considerando marcos conceptuales de la literatura de Fl de una metodología y se tuvo en cuenta la evaluación de los componentes críticos del proyecto. 
programa (Hohmann \& Shear, 2002). Además se reconoce su potencial para monitorear y mejorar la aplicación durante la misma aplicación (Century et al., 2010). Por esa razón, se entiende que, si hay varias mediciones de $\mathrm{Fl}$ en una intervención, los resultados de estas puedan ir aumentando.

Ahora bien, la validez externa también aumenta al proporcionar guías y evidencias útiles para la replicación del programa que se espera medir (Mowbray et al., 2003). Al respecto, en un análisis simple de dos dimensiones de una intervención -resultados (positivos o negativos) y la fidelidad observada (alta o baja) - se genera una tabla de análisis que permite ponderar la utilidad del escalamiento de la intervención a otras esferas y alcances (Tabla 1).

Tabla 1.

Visualización del análisis

\begin{tabular}{|l|l|c|}
\cline { 2 - 3 } Resultados positivos & No escalar & Escalar el proyecto \\
\cline { 2 - 3 } Resultados negativos & No escalar & No escalar \\
\cline { 2 - 3 } & Baja fidelidad & Alta fidelidad
\end{tabular}

Fuente: Duyar (2006, en O'Donnell, 2008)

La conclusión simple de este análisis radica en que convendría escalar proyectos que logren resultados positivos y evidencian alta fidelidad; de lo contrario, se pueden hacer inversiones en programas que gestionen prioridades que no tengan que ver con los resultados (de alta fidelidad, aunque negativos) o que produzcan un falso positivo concerniente a la intervención (resultados positivos, aunque de baja fidelidad en la implementación). Por supuesto, esta simple regla no siempre se aplica de forma automática, y existen detractores de su aplicación lineal en contextos escolares (Fullan, 1983; Reid, Hopkins y Holly, 1987).

También se evidenció que la FI incrementa la validez estadística de las investigaciones que miden intervenciones y se conforma como variable moderadora, lo que explica parte de los efectos resultantes (Teague, Drake \& Ackerson, 1995; Dumas, Lynch, Laughlin, Smith y Prinz, 2001; Mowbray et al., 2003). Por tanto, es posible así precisar cuántos de los resultados de una intervención pudieron fallar por faltas de implementación o por la pobreza del diseño inicial (Summerfelt, 2003).

Esta indistinción, producto del enfoque de la $\mathrm{Fl}$, se perpetúa como uno de los puntos más discutibles de este enfoque. La principal limitante que se discute es la incertidumbre frente a la pobreza del diseño y a la mejora de la implementación, aun cuando el modelo de evaluación sea sólido. La analogía, "conocer un problema no significa que podamos resolverlo" sirve para explicar que una buena medición de la fidelidad no significa que active las capacidades necesarias para mejorar la intervención. Sin embargo, si se reporta información sobre el proceso que se está llevando a cabo es más probable reaccionar y tomar decisiones de mejora del proyecto en marcha (Century et al., 2010; O’Donnel, 2008). En otras palabras, la información brindada por la Fl no asegura mejores decisiones y capacidades, pero sí las posibilita.

En oposición a la Fl, se postulan estrategias de implementación de innovaciones escolares (usualmente curriculares) que enfatizan en un enfoque 
adaptativo-evolutivo que contrasta los diseños a priori con modificaciones a lo largo del proceso (Altrichter, 2005). Las razones para preferir este enfoque no obedecen solo a las limitantes circunstanciales, sino más bien a los conocimientos situados que tienen los agentes que implementan y/o emergen en las innovaciones mismas. Este enfoque pone el énfasis de la agencia en la dimensión interna de la organización y amplía la observación de efectos, detectando por ejemplo los colaterales (Schön, 1983). En otras palabras, la predefinición de criterios de observación para un proyecto impone una limitante a los resultados que se pueden encontrar. Al seguir una estrategia más adaptativa, esta evaluación se amplía y permite incorporar elementos que no se habían considerado. Sin embargo, este enfoque también acarrea problemas, al plantear objetivos y criterios de evaluación de forma ambigua, ya que es más sensible a la percepción de éxito, que varía según las personas y los casos (Fullan, 1983). Por otra parte, el mismo autor indica que el enfoque programado de la Fl es más adecuado, si el cambio es pequeño y gradual, con sistemas de evaluación y enseñanza previamente conocidos, y si los casos son estables.

Metodológicamente, la Fl se construye con base en "criterios de fidelidad" que operacionalizan conceptos centrales del diseño del programa evaluado. Si bien la literatura discute los criterios, se han consensuado algunas distinciones en meta-análisis e investigaciones de casos que se expondrán a continuación. Una convergencia relevante consiste en que la FI se debe poder medir ampliamente para cubrir la variedad de diseños posibles, pero debe contener elementos centrales o "conceptos críticos" de lo que específicamente se hará para construir indicadores (Century et al., 2010).

Una primera distinción frecuente consiste en que hay componentes críticos de fidelidad de la estructura que se constituyen como condiciones necesarias para que se lleve a cabo la intervención o programa (por ejemplo, frecuencia, tipo de sesiones, disponibilidad de personas o capacidades de ejecutar una tarea) y conceptos críticos de proceso que reportan la forma en la que se lleva a cabo el programa (por ejemplo, diferencias con otros programas, calidad de las acciones, respuesta de los participantes) (Mowbray et al., 2003; Century et al., 2010).

Aparentemente, la fidelidad de un determinado programa es más probable cuando existe un "manual de intervención" que defina con claridad y articule con teorías los componentes críticos (O’Donnell, 2008). La divergencia encontrada radica en que diferentes investigadores no comparten modelos de intervención ni coinciden en lo que sea la implementación fiel, lo que hace aún más interesante este campo en educación (Century et al., 2010).

Las convergencias radican en la operacionalización, en la que se propone aprovechar un marco conceptual ampliamente recomendado para estructurar los criterios de fidelidad y ubicar los componentes críticos (Century et al., 2010), mientras que las mediciones se debieran cuantificar para poder relacionarlas con resultados (O'Donnell, 2008). Las dimensiones que se rescatan (exposición, entrenamiento, calidad instruccional, respuesta y diferenciación) provienen de previas discusiones en la literatura, y se detallan a continuación.

Exposición: a menudo se homologa con la dosificación, concepto que determina la frecuencia, tiempo y duración que se considera para la implementación. Esta exposición puede variar según los participantes y el tipo de instancia en la que se los expone al material y/o contenidos. Este sería el diseño estructural de la intervención.

Entrenamiento: esta dimensión integra todo lo que impacte sobre la capacidad de los participantes para realizar la tarea intencionada. 
En algunos casos, son actividades de aprendizaje; en otros, puede tratarse de condiciones ya dadas por la formación previa o incluso de la organización de equipos o de recursos como tiempo o sueldos destinados a una función. De ahí que los componentes puedan exceder el nombre "entrenamiento", en referencia a una estructura organizacional que da soporte y sostiene capacidades.

Calidad Instruccional: se adentra en los componentes de proceso, definiendo mediante estándares o constructos lo que debe suceder en la intervención. Puede tratarse de experticias, acciones, valores o afectos que llevan a la práctica los participantes.

Respuesta: siempre se refiere a las reacciones de los participantes, en las que usualmente se reporta la participación, asistencia o entusiasmo. Los componentes de esta dimensión corresponden al grado de asimilación y uso de la propuesta de intervención por parte de estos.

Este marco conceptual resume gran parte de los hallazgos sobre la discusión en $\mathrm{Fl}$ en educación y considera dimensiones más amplias que no se trataron mayormente en esta investigación, como la diferenciación entre programas, la adherencia o las limitantes encontradas entre estudios (Century et al., 2010), además de las objeciones a enfoques tradicionales de implementación versus enfoques más adaptativos y situados (Fullan, 1983; Reid et al., 1987).

\section{Liderazgo instruccional}

El modelo de liderazgo llamado "instruccional" es parte de los diferentes enfoques de liderazgo escolar o pedagógico que se han desarrollado desde hace más de 30 años. Si bien comparte las características generales de definirse como una capacidad de influencia social con objetivos organizacionales, se precisa como una priorización en la calidad de la enseñanza y el logro de aprendizajes que alinea y motiva a los miembros de la organización hacia la mejora escolar (Hallinger \& Heck, 2002). Se desmarca de otros modelos usados en la investigación del liderazgo que se enfocan principalmente en la interacción motivadora, inspiracional y transformacional (Leithwood, 1992) o de los enfoques centrados en distribuir la influencia entre agentes de la escuela (Spillane, Halverson \& Diamond, 2004). Aun así, es muy importante que el modelo de liderazgo instruccional no se entienda como un enfoque excluyente de estos énfasis, sino más bien como foco complementario e integrado al fenómeno global del liderazgo escolar.

De este modo, el liderazgo instruccional se asocia a prácticas específicas y a dinámicas de influencia que contribuyen a la estabilidad, la calidad de la enseñanza y el mejoramiento de logros de aprendizaje de los estudiantes (Robinson, Lloyd y Rowe, 2008; Hallinger y Murphy, 1985; Marzano, Waters y Brian, 2005; Leithwood, Day, Sammons, Harris y Hopkins, 2006). En este contexto conviene representar el liderazgo instruccional como una red de relaciones que genera coinfluencias de agentes y prácticas, las cuales se distribuyen en el sistema, la organización y las interacciones entre los involucrados (Volante, 2010).

Más que un fenómeno de aportes individualizados, la idea de red implica prácticas que consisten en generar condiciones y estimular la acción de otros (Leithwood y Riehl, 2005). Esta idea también coincide con las líneas de liderazgo distribuido (Harris, 2009; Spillane, Camburn y Pareja, 2007), atingentes a que el ejercicio del liderazgo escolar supone distribuir la influencia y converger en los objetivos académicos compartidos. Por ello, se ha planteado que la percepción de mayor empoderamiento entre 
profesores -quienes se involucran en la distribución de poder- se relacionaría con los resultados académicos, la percepción de eficacia colectiva y la confianza organizacional, como claves de la efectividad escolar (Hoy, 2002).

Estas características de la función del liderazgo escolar son coherentes con los antecedentes teóricos sobre la influencia indirecta de los directores en los logros educativos, pues supone que ellos actúan a través de otros para ejercer influencia en profesores, padres y estudiantes. Esto implica que los directivos eficaces han logrado equilibrar las diversas demandas e involucrar a los distintos agentes, balanceando sus intereses y articulando una diversidad de objetivos institucionales (Volante, 2010).

La influencia instruccional se ejerce en diversas dimensiones, como las que implican lograr una visión conjunta, el desarrollo del clima de aprendizaje y la gestión del programa instruccional (Hallinger \& Murphy, 1985). Un ambito específico de influencia ha sido la enseñanza y el aprendizaje en matemáticas, mediante la instalación de prácticas sistemáticas que alinean a directivos, profesores y estudiantes, en funcion de metas e innovaciones en la forma de coordinar, implementar y monitorear prácticas de ensenanza y gestión académica en matemáticas (Heck \& Hallinger, 2009). En consecuencia, esta lógica de muestreo en matemáticas y la observación de equipos multinivel fundamentan el proyecto de intervención en el que se inserta este estudio (Volante, 2011).

\section{Antecedentes del proyecto}

El proyecto Fondecyt No. 11100466 en el cual se enmarca esta investigación se propuso desarrollar equipos de liderazgo instruccional (ELI) en seis escuelas, con un grupo de 24 directivos y profesores y una cohorte de más 500 estudiantes. El objetivo de la intervención fue mejorar la gestión académica y los resultados de aprendizaje en matemáticas, entre el año 2010 y 2012, período en que los estudiantes finalizaban el $10^{\circ}$ año de escolaridad en Chile (año II de secundaria). El desarrollo de estos equipos de liderazgo instruccional se facilitó por medio de una intervención del equipo de investigadores que asistió a los colegios, con el fin de proporcionar un conjunto de prácticas para ser aplicadas y capacitar a los equipos en talleres grupales con todos los colegios.

Considerando la idea de "componentes críticos" (Century et al., 2010), en el proyecto se puso especial énfasis en su definición, pues ellos determinan el estándar con el que se mide la fidelidad. Por tanto, se recomienda su extracción desde las fuentes escritas que proporcionan los interventores (Leithwood \& Montgomery, 1980; Century et al., 2010), en este caso se anazalizó el Manual de Prácticas de Influencia Instruccional confeccionado a la luz de los antecedentes sistematizados por el equipo de investigadores, en el cual se definieron siete prácticas, con ejemplos concretos para llevarlas a cabo:

Práctica 1. Definir qué se entiende por una buena enseñanza de la matemática: Se esperaba que los miembros del ELI determinaran estándares de desempeño de los docentes y de contrastación para los directivos. El ejemplo concreto entregado por el equipo fue una pauta de observación de clases adaptada de la "Administrators Guide: How to support and improve Mathematics Education in your School" (2003), del libro The principal as Mathematics Leader (Ontario Principal's Council, 2009), a partir de la cual cada equipo escolar iba desarrollando su propio modelo para precisar las prácticas aludidas.

Práctica 2. Definir los aprendizajes clave para matemáticas en $2^{\circ}$ medio: Los integrantes de cada ELI debían consensuar qué elementos del currículo nacional serían seleccionados como prioritarios, para asignarles horas lectivas, ser evaluados y secuenciarlos a lo largo del año. En los talleres presenciales y con reforzamiento en terreno se entregaron criterios (como la necesidad de contar con el aprendizaje en el futuro u otras asignaturas) e incluso se precisaron recomendaciones para esta definición. El resultado diferenciaba niveles específicos, como el de aprendizaje clave consistente en establecer relaciones entre los logaritmos, potencias y raíces, y otros aprendizajes esperados, sin que fueran clave, como el de deducir propiedades de los logaritmos.

Práctica 3. Llevar a los estudiantes a que se pongan metas: Se esperaba concretar actividades en las que cada estudiante tuviera la oportunidad de formular metas para su desempeño durante el año escolar. Estas metas debían ser cuantificables y voluntarias, excluyendo estándares impuestos por la institución o el profesor. La recomendación del equipo se concretó en un formulario que pedía, entre otras, calificaciones esperadas para cada semestre que cursaría el estudiante o también su predicción en el puntaje de la prueba externa que se aplicaba en el colegio.

Práctica 4. Asegurar que todos los estudiantes tengan experiencias de éxito en matemática: Al implementar esta práctica, los participantes debían diseñar un método mediante el cual cada estudiante podía demostrar avance en el aprendizaje, con un efecto de autorregulación respecto a sus capacidades y estrategias, como también un efecto sobre su autoestima, al sentirse competente en la tarea encomendada. El equipo incentivó la implementación de Desafíos de aprendizaje con la siguiente metodología. Se entregaba repetidamente durante el año un set de ejercicios que cubría todo el currículo en tres niveles de dificultad 
(alto, intermedio y bajo). La aplicación de estas "pruebas" no se calificaba y se reforzaba de nuevo en clases enfocadas a los aprendizajes a los que apuntaban (definidos como claves). Luego se realizó una segunda aplicación en la que los estudiantes podían demostrar su aprendizaje en los mismos problemas.

Práctica 5. Monitorear metas curriculares y de los estudiantes: Mediante este monitoreo se esperaba que los equipos tuvieran constante información sobre los avances y atrasos curriculares, desempeños y cumplimientos de metas personales. Para ello se proporcionaron dos instancias de una evaluación estandarizada: una prueba externa (Sistema de Evaluación de Progreso del Aprendizaje - SEPA) y los "desafíos" ya descritos.

Práctica 6. Reforzar a estudiantes débiles y aventajados: Los miembros de los equipos debían implementar prácticas en las cuales se hiciera un trabajo diferenciado según el nivel de los estudiantes, tanto fuera del aula, con talleres o reforzamientos, como dentro de ella. Para ello se entregaron guías que comprendían ejercicios con crecientes niveles de dificultad para que cada estudiante pudiera ajustarse.

Práctica 7. Realizar ciclos de observación y retroalimentación acerca de iniciativas de mejora: Los participantes eran inducidos a buscar evidencias que permitieran retroalimentar sus avances y plantearse mejoras, por lo que los investigadores capacitaban en terreno para la aplicación de "caminatas de aula", ejercicios de retroalimentación que consisten en realizar observaciones breves (7 min. aprox.) y focalizadas en el trabajo que realizan los estudiantes en clases seleccionadas, las cuales se registran como evidencias que incentivan a la reflexión, la evaluación de avance e iniciativas de mejora.

Finalmente, más allá de las siete prácticas clave, el equipo de investigación proporcionaba retroalimentación permanente, en diferentes instancias que permiten un aprendizaje y monitoreo de los modos de adopción e innovación en cada establecimiento. Esto permite contextualizar el enfoque de intervención de estas siete prácticas y generar información más amplia que favorece el aprendizaje y análisis situado caso a caso.

\section{Metodología}

\section{Problema de investigación}

El problema general de un estudio de fidelidad de implementación consiste en capturar y procesar evidencia que permita afirmar en qué grado se cumplen los términos propuestos en un determinado diseño de intervención. En este caso, el problema se centra específicamente en el grado en que los participantes de los equipos de liderazgo instruccional (ELI) en efecto implementan las prácticas y en cómo se caracteriza en cada contexto escolar. Para ello, se recolectaron datos para describir el grado en que se cumplen las cuatro dimensiones de fidelidad de implementación (Century et al., 2010) en los seis establecimientos participantes. A continuación se presentan los descriptores de las cuatro dimensiones de Fl aplicadas a la intervención estudiada:

Exposición: Se esperaba que cada escuela participara de cinco "talleres" de diseminación e intercambio de prácticas, con una periodicidad mensual, y recibiera un total de siete "visitas en terreno" durante un año. La duración de los talleres fue de 3 horas, y las visitas fluctuaban entre 1,5 y 2 horas. Los datos fueron completados directamente por el equipo de investigación, en la medida que algún participante ELI asistiera al taller, y se concretaban las visitas programadas.

Entrenamiento: La unidad básica de intervención es la conformación de equipos de liderazgo instruccional, compuestos por el director del establecimiento, el responsable de la coordinación académica (en Chile, Jefe de Unidad Técnico Pedagógica) y el o los profesores de matemáticas que enseñaban en la cohorte 2010-2012 de estudiantes de $9^{\circ}$ y $10^{\circ}$ grados. También se consideró la participación de profesores de otros niveles de matemáticas o de ciencias, por los efectos positivos que reporta el trabajo colegiado. Por tanto, este indicador lo conformó el equipo que participaba en las actividades y visitas en cada establecimiento.

Calidad instruccional: Esta dimensión se evaluó con una rúbrica comparativa diseñada según juicio experto. En ella, para cada una de las siete prácticas se especificaban elementos relacionados con cumplir o no cumplir con lo esperado. Por ejemplo, la Práctica 2 de acordar aprendizajes clave requería un acuerdo grupal, referentes externos (como materiales oficiales) y la explicitación de criterios de dificultad o secuenciación, para considerarla fielmente cumplida. La rúbrica permitió discriminar entre los seis casos señalando cumplimientos mayores y menores, con gradaciones. El instrumento requirió de información amplia y consultada para captar el proceso en el tiempo y las adaptaciones que los participantes hicieron de las propuestas del programa a sus contextos.

Respuesta: Esta dimensión se constituyó, por un lado, con los registros de asistencia y participación de los equipos a los talleres y actividades y, por el otro, con los efectos positivos reportados por los participantes, como mejora en las condiciones laborales, replicación de la intervención o satisfacción de los alumnos, entre otros. La asistencia y los reportes fueron considerados por partes iguales como indicadores de motivación, asimilación y uso por parte de los participantes.

En síntesis, al evaluar las cuatro dimensiones expuestas, se aprecia el grado de implementación de la intervención. El nivel de consistencia de cada caso 
intervenido se refleja en los puntajes, con escala de 0-1, elegida por la simpleza en el cruce de variables y efectos que se puedan reportar directamente con uso de proporciones.

Al comparar los seis establecimientos intervenidos, se sistematiza la apreciación de los interventores, contrastando las evaluaciones implícitas que se generan en las interacciones de talleres, visitas y monitoreo con el cumplimiento de las acciones clave requeridas en el enfoque de intervención. De este modo, se lanza la hipótesis de que la Fl que logra cada colegio mantendrá una relación con los resultados en las mediciones de motivación y el logro matemático, que compromete la intervención a tres años.

\section{Muestra}

Para explicitar algunas características del contexto en que se realiza el proyecto de intervención y este estudio de fidelidad de la implementación, es necesario señalar que, de una muestra de doce establecimientos, aleatoriamente se asignaron seis a una condición experimental y otros seis a una de control. Todos los casos fueron extraídos del universo de colegios que tienen características socioeconómicas y de rendimiento en el promedio nacional. Con ello se utilizó un muestreo de casos equivalentes, que presumiblemente son afectados por igual por otras variables intervinientes, como el efecto hogar. Se considera así un doble criterio de selección respecto a la paridad y la cercanía con el promedio nacional obtenido en las pruebas de aprendizaje estandarizadas y aplicadas por el Ministerio de Educación de Chile (Pruebas Simce 2010), además se confía en la representatividad teórica de esta muestra respecto a la paridad con el promedio nacional en la escala de nivel socioeconómico y tamaño de la escuela.

\section{Instrumento}

Tal como se ha indicado, las pautas y rúbricas para medir la implementación las desarrolló el equipo de investigación, pero no todos sus miembros estaban dedicados a la intervención directa con los participantes. La aplicación de estos instrumentos se realizó en tres etapas, en el período de marzo y mayo de 2012:

Primera etapa: contempló la presencia de todos los participantes del equipo de liderazgo instruccional (ELI) para comentar preguntas y descriptores que ejemplificaran de forma abierta las acciones y situaciones que consideraron pertinentes para cada práctica propuesta. En este sentido, cuando se procedió a indagar por una práctica específica, el entrevistador no sesgó la pregunta a una respuesta ligada al descriptor propuesto en el manual de prácticas del proyecto, sino que indujo a describir "lo que se hace" para lograr lo propuesto en una determinada práctica.

Segunda etapa: en esta se organizaba a los entrevistados según sus cargos y se les solicitaba que consignaran esta información en un nuevo formulario, para diferenciar a cada integrante del equipo ELI entre directivos o profesores. Con esto se buscó un reporte más preciso y un ejemplo para observar la recomendación del manual y la práctica en general. Por ejemplo, en la Práctica 6: Reforzar a estudiantes con dominio débil y avanzado en matemáticas, se pidió al profesor describir el material de clases y sus actividades respecto al trabajo diferenciado con alumnos rezagados y/o adelantados y a los directivos, describir los resultados que se han obtenido en las iniciativas con los estudiantes con dominio débil y/o avanzado de matemáticas. Esta diferencia por cargos, como se aprecia en el ejemplo, se centra en los aspectos de ejecución, para los profesores, y de seguimiento, para los directivos. Estos datos fueron registrados por escrito por cada participante y luego clasificados e integrados al informe. 
Tercera etapa: por ser de validación, se realizó sobre la base de una propuesta de preinforme confeccionada por miembros del equipo de investigación, que, al entrevistarse con los participantes del ELI, pudieron complementar, precisar y rectificar información. En ningún caso fue necesario eliminar información que se hubiera reportado en cualquiera de las dos fases previas, y más bien se la contextualizó y se detallaron descripciones. Con ello se procedió a terminar los informes definitivos sobre cada práctica, que fueron usados como insumos para el análisis que se presenta en esta publicación.

\section{Plan de análisis}

Se presentan a continuación los procedimientos para construir los datos que se presentan en los resultados. La dimensión de la exposición se determinaba según el porcentaje total de talleres a los que asistió algún miembro de los ELI, promediado con la proporción de visitas que llevaron a cabo en las escuelas miembros del equipo de investigación:

Figura 1.

Fórmula de exposición

Exposición $=\quad \begin{gathered}\begin{array}{c}\text { \# de talleres a los } \\ \text { que assitó algún } \\ \text { miembro del ELI }\end{array} \\ \begin{array}{c}\text { \# de talleres } \\ \text { diseñados (5) }\end{array}\end{gathered}$

Fuente: Elaboración propia

Entrenamiento: esta dimensión reporta un punto completo para cada uno de los tres niveles de responsabilidad de los participantes ELI: directores, mandos intermedios y profesores. Por ello pueden darse valores de 0 , en caso que un estamento no participe, o de 0,5, si solo participa un docente de los dos cursos. Como otros docentes son considerados elementos favorables, los casos ELI que incluyan profesores de otros subsectores o de otros niveles entre sus actividades de aprendizaje e implementación agregan 1 a los valores promediados, como posible medida de compensación frente a otros agentes ausentes. Para que el promedio mantenga la escala, el denominador aumenta de 3 a 4, dependiendo si se puntúan 3 ó 4 estamentos profesionales:

Figura 2.

Fórmula de entrenamiento

$\begin{array}{ll} & +1 \text { si integra director } \\ & +1 \text { si integra jefe técnico } \\ \text { Entrenamiento }=\quad & +1 \text { si integra } 2 \text { profesores de la cohorte } \\ & +0,5 \text { si integra } 1 \text { de los profesores de la cohorte } \\ & +1 \text { si integra } 1 \text { de los profesores adicionales } \\ & \text { (otros niveles o asignaturas) }\end{array}$

3 ( o 4 si integran profesores adicionales) 
Calidad: esta dimensión se basa en la rúbrica de la Pauta de Seguimiento y contempla hasta cinco criterios de logro, que suman un punto cada uno. Se distinguen, entre estos, siempre tres criterios centrales (necesarios para la práctica fielmente cumplida) y dos adicionales (deseables, pero no necesarios) que modifican el denominador del promedio. Por ejemplo, el caso 3 en la Práctica 1 cumple con dos criterios centrales ("Formalizan la buena enseñanza con criterios para el trabajo en sala u otras funciones" y "Los profesores y directivos acceden, usan y comparten entre sí estos criterios") y no cumple con el otro ("Se revisan en conjunto los resultados y efectos que tiene la enseñanza promovida"). Por tanto, suma 2 puntos y se promedia por 3, lo que arroja el puntaje 0,67.

A su vez, hay criterios adicionales que agregan un punto completo, pero producen un efecto diferenciador marginal frente a los criterios centrales, al aumentar el denominador con el que se calcula el promedio. Por ejemplo, el caso 3 en la Práctica 2 cumple nuevamente con dos criterios centrales ("Los aprendizajes son construidos, decididos y discutidos grupalmente" y "Aplican en la elección de aprendizajes clave referentes externos"), no cumple con el otro ("Aplican en la elección de aprendizajes clave criterios técnicos de secuenciación, dificultad creciente, habilidades requisito o uso en otras asignaturas"), pero esta vez sí cumple con un criterio adicional: "Comparan la cobertura curricular entre cursos (ejemplo: pruebas de nivel)". Esta vez se suman 3 puntos, pero aumenta también el denominador de 3 a 4 (por un criterio adicional) y con ello se obtiene el puntaje 0,75.

Cada conjunto de indicadores arroja el puntaje de una práctica y las siete prácticas promediadas el puntaje de calidad total:

Figura 3.

Fórmula de calidad

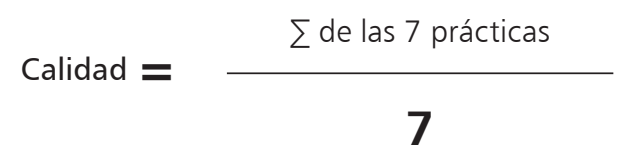

$\sum$ de los criterios cumplidos en la rúbrica

(+1 por cada criterio)

Cada práctica

3 (+1 por cada criterio adicional de la rúbrica) que se cumplía

Fuente: Elaboración propia

Respuesta: finalmente, esta dimensión se construye según una lista de categorías que reportaron los mismos participantes del ELI y evidenció la asistencia de los individuos. Cuando se reporta algún efecto dentro de una de las seis categorías, se asigna un punto completo de 1, y la suma de las categorías logradas se divide por el total de categorías. Este puntaje de efectos reportados se promedia con la asistencia de los participantes a los talleres, como medida de la adherencia y motivación individual, que por cierto se distingue de la participación de los ELI (de cualquier integrante) como instancias de la dimensión entrenamiento: 
Figura 4.

Fórmula de respuesta

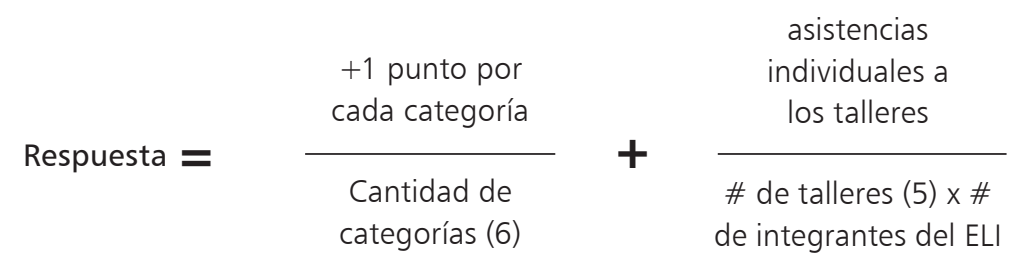

2

Fuente: Elaboración propia

Validez,

Es importante en este punto señalar que en este estudio solamente se ha trabajado con reportes de los miembros de los equipos sobre sus prácticas y aún no se emplean observaciones directas en los establecimientos. Con ello es claro que hay limitaciones en la contrastación de información y, eventualmente, sesgos de autoafirmación en equipos que predeciblemente favorecen el nivel de implementación de sus colegios. Sin duda, esto puede ser una limitante a la validez por reactividad de parte de los equipos que ven evaluadas sus propias prácticas. Sin embargo, dado que este efecto se da en todos los casos de este estudio, los datos mantienen su comparabilidad y similitud de condiciones.

En consideración a estas limitaciones, este estudio ofrece los primeros parámetros para el diseño y evaluación de fidelidad en el contexto de intervenciones que inducen el ejercicio del liderazgo instruccional en establecimientos escolares. Esta misma ya está siendo complementada con estudios de observación en los casos intervenidos en el año 2012, y en una siguiente etapa se espera utilizar resultados en pruebas de rendimiento nacionales como otra fuente de evaluación. Se puede definir entonces esta investigación como una primera exploración sobre fidelidad de implementación aplicada a una intervención específica sobre influencia instruccional en la motivación y rendimiento en estudiantes secundarios.

\section{Resultados}

En este segmento se presentan los resultados de la medición de fidelidad de implementación según los objetivos de la investigación, y se recapitula sobre ellos:

- Reportar los niveles de FI del proyecto Equipos de Liderazgo Instruccional.

- Diferenciar los niveles de Fl que se observen en los seis colegios participantes de la intervención del proyecto.

El primer objetivo refiere a los datos generales de fidelidad según el marco conceptual que ya ha sido expuesto. Principalmente corresponde al promedio de las evaluaciones específicas en cada una de las cuatro dimensiones o componentes críticos. 
Figura 5.

Fidelidad de la implementación (FI) de cada componente crítico

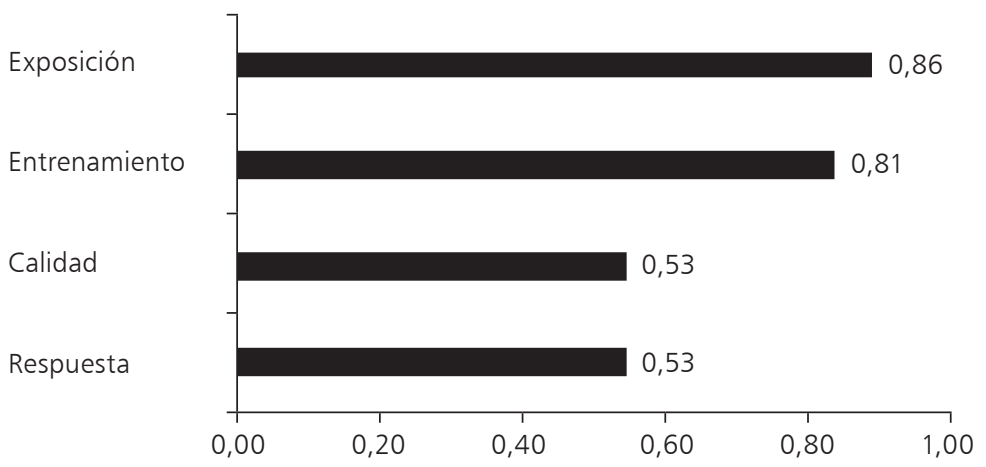

Fuente: Elaboración propia

Estos datos reflejan una fidelidad mucho más alta en los componentes estructurales (exposición y entrenamiento) que en los de proceso (calidad y respuesta). Las desviaciones estándar fueron de 0,14, 0,16, 0,06 y 0,15 en orden respectivo, lo que indica una mayor estabilidad en los datos de los diferentes casos sobre calidad. Como estos datos son proporciones entre 0 y 1 , se puede igualar conceptualmente a proporciones que expresan porcentajes de cumplimiento de los propósitos iniciales. El resultado aborda las siete prácticas que sustentan el proyecto ELI para poder comparar cuáles fueron llevadas a la práctica con más fidelidad que otras.

Figura 6.

Fidelidad de la implementación (FI) de cada práctica

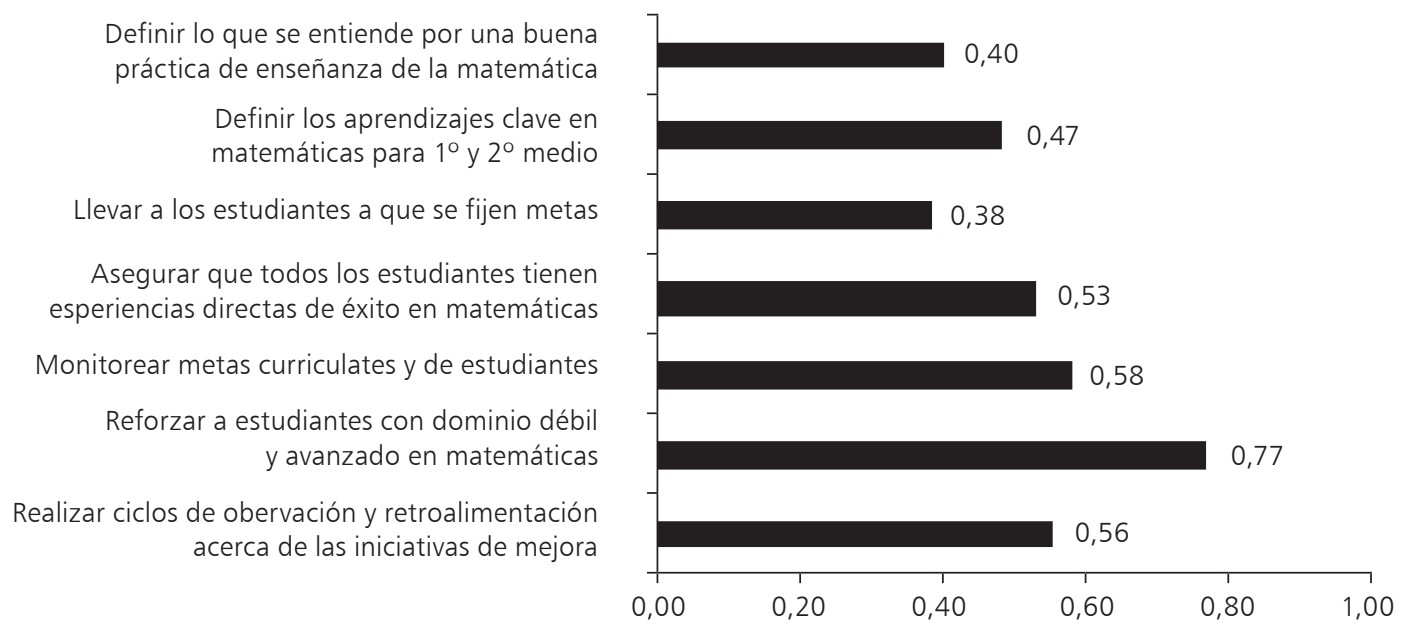

Fuente: Elaboración propia

Los datos indican que los participantes de los ELI tuvieron mayor capacidad de llevar a la práctica los reforzamientos, el monitoreo de metas y los ciclos de observación que para realizar las definiciones de aprendizajes clave, las definiciones de buena enseñanza o un proceso en el que los estudiantes fijen metas para sí mismos. Finalmente, se hace relevante presentar los seis casos, de modo que se pudiera diferenciar la fidelidad 
de la implementación en cada uno. Para fines comparativos, se incluye un promedio entre los cuatro componentes críticos (que conceptualmente no guarda mayor sentido) y luego se presenta un desglose por práctica.

Figura 7.

Fidelidad de la implementación (FI) de cada caso

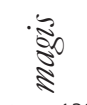

PÁGINA 130

$\stackrel{\text { m }}{\stackrel{5}{2}}$

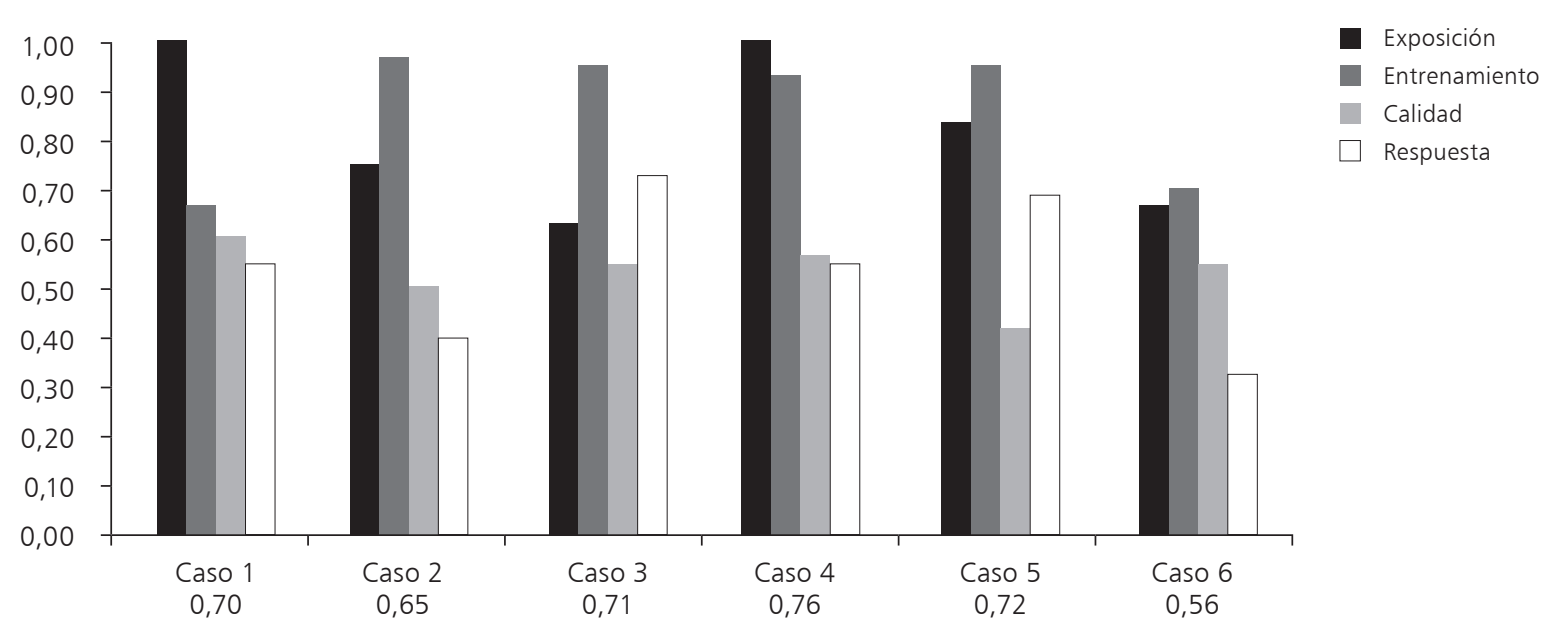

Fuente: Elaboración propia

En estos datos pareciera ser particularmente pertinente el hallazgo de que las dimensiones estructurales (exposición y entrenamiento) no parecen relacionarse con la dimensión de calidad, que se mantiene más estable entre los casos. Por otro lado, la dimensión respuesta parece variar más acorde a la Calidad. También se puede notar en los promedios que figuran bajo la etiqueta del caso que hay una relativa paridad, con excepción del caso 6 y, en menor medida, del caso 2.

Si ahora se comparan los seis casos según los resultados en las siete prácticas, se obtiene el gráfico de la Figura 8.

Figura 8.

Fidelidad de la implementación (FI) de las prácticas según el caso

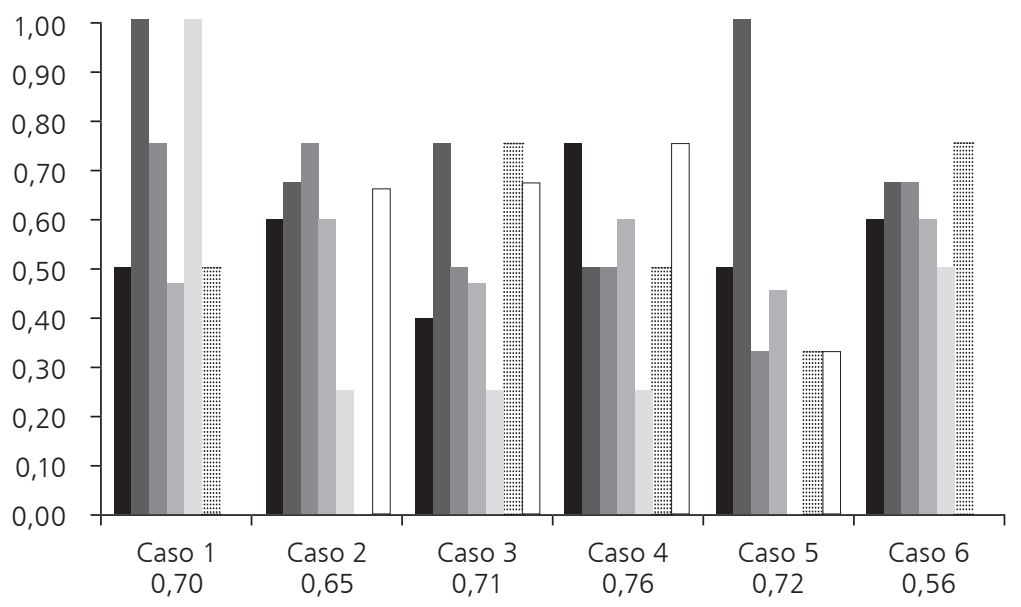

Definir lo que se entiende por una buena práctica de enseñanza de la matemática

- Definir los aprendizajes clave en matemáticas para $1^{\circ}$ y $2^{\circ}$ medio

- Llevar a los estudiantes a que se fijen metas

- Asegurar que todos los estudiantes tienen esperiencias directas de éxito en matemáticas

Monitorear metas curriculates y de estudiantes

Reforzar a estudiantes con dominio débil y avanzado en matemáticas

$\square$ Realizar ciclos de obervación y retroalimentación acerca de las iniciativas de mejora

Fuente: Elaboración propia 
De estos resultados se puede inferir que la fidelidad de la implementación total no necesariamente tiene que ver con la calidad de las prácticas implementadas, pues se notan casos, como el 5 y el 6 , en los cuales esa relación está invertida. Tampoco parece haber un patrón típico de conformación de calidad entre los diferentes casos, lo que parecía pasar con la visión total sobre las prácticas, que podría haber hecho pensar que la forma de llevar a la práctica la intervención fuera parecida entre casos.

Para mayor claridad se exponen en la Tabla 2 los promedios completos obtenidos en esta fase de la investigación:

Tabla 2.

Resumen de resultados

\begin{tabular}{c|c|c|c|c|c|c|c}
\hline Caso & $\mathbf{1}$ & $\mathbf{2}$ & $\mathbf{3}$ & $\mathbf{4}$ & $\mathbf{5}$ & $\mathbf{6}$ & \\
\hline Promedio & 0,70 & 0,65 & 0,71 & 0,76 & 0,72 & 0,56 & Fl total: \\
\hline Exposición & 0,67 & 0,96 & 0,95 & 0,93 & 0,95 & 0,71 & \\
\hline Entrenamiento & 1,00 & 0,75 & 0,63 & 1,00 & 0,83 & 0,67 & \\
\hline Calidad & 0,60 & 0,51 & 0,54 & 0,55 & 0,42 & 0,54 & \\
\hline Respuesta & 0,54 & 0,40 & 0,72 & 0,55 & 0,68 & 0,33 & \\
\hline Prácticas & & & & & & & Promedio \\
\hline $\mathbf{1}$ & 0,00 & 0,67 & 0,67 & 0,75 & 0,33 & 0,00 & 0,40 \\
\hline $\mathbf{2}$ & 0,50 & 0,00 & 0,75 & 0,50 & 0,33 & 0,75 & 0,47 \\
\hline $\mathbf{3}$ & 1,00 & 0,25 & 0,25 & 0,25 & 0,00 & 0,50 & 0,38 \\
\hline $\mathbf{4}$ & 0,46 & 0,60 & 0,46 & 0,60 & 0,46 & 0,60 & 0,53 \\
\hline $\mathbf{5}$ & 0,75 & 0,75 & 0,50 & 0,50 & 0,33 & 0,67 & 0,58 \\
\hline $\mathbf{6}$ & 1,00 & 0,67 & 0,75 & 0,50 & 1,00 & 0,67 & 0,77 \\
\hline $\mathbf{7}$ & 0,50 & 0,60 & 0,40 & 0,75 & 0,50 & 0,60 & 0,56 \\
\hline \hline
\end{tabular}

Fuente: Elaboración propia

\section{Discusión de resultados}

De acuerdo con los resultados expuestos, es posible sintetizar en cuatro afirmaciones la evaluación preliminar de la fidelidad de implementación (FI) de prácticas de liderazgo instruccional, en el contexto del estudio que busca transferir e impactar en resultados educativos de seis establecimientos secundarios de la región metropolitana en Chile:

La fidelidad de los componentes estructurales fue mayor que la fidelidad de los de proceso: considerando que la estructura estaba determinada por la adherencia de los equipos a las instancias de intervención y a su conformación en los tres estamentos jerárquicos y por posible apoyo de otros docentes, se puede pensar que la gestión del tiempo y la disponibilidad de las personas en las escuelas es una tarea mejor realizada. Esto se condice con los antecedentes que describen redes sociales de influencia (Volante, 2010). Sin embargo, la influencia instruccional como tal y el foco en el proceso de enseñanza-aprendizaje, que se refleja en las dimensiones de proceso, se ven dificultadas en los casos. Intervenciones como esta, que fortalecen la labor instruccional, se justifican por atender lo que parece ser un debilidad de la gestión escolar, con consecuencias relevantes (Marzano et al., 2005; Heck y Hallinger, 2009).

La práctica que busca que los estudiantes se fijen metas propias resultó ser la más disminuida: la hipótesis que sustenta esta práctica busca efectos de motivación en estudiantes secundarios, desde la influencia de 
directivos y docentes. Si se desglosan los logros de cada caso en la práctica 3 , se observa que solo el caso uno tuvo un logro alto, mientras que en los demás se detectaron logros bajos, nulos o de la mitad de lo esperado. Esto permitió buscar remediales durante el proceso del 2012, apelando al buen ejemplo del primer caso, en función de mejorar el desempeño de los otros. Esta es una de las principales ventajas que ofrece la $\mathrm{Fl}$, al monitorear la aplicación durante la aplicación misma (Century et al., 2010).

La práctica de reforzar a los estudiantes según su nivel es la fortaleza de los colegios estudiados: este hallazgo, para la gestión de las escuelas significa una preocupación por los resultados de sus estudiantes. Si además se considera el buen nivel de las acciones de monitoreo de logros de objetivos y de reflexiones para la mejora, se refuerza esta idea. El que este perfil sea además más estable entre las escuelas que en las prácticas con resultados más bajos radica en funciones de control mejor desarrolladas. Esto constituye otro argumento para priorizar los aspectos más instruccionales en estos colegios, ya que se facilita la focalización y concentración en este tipo de objetivos.

Si bien hay un nivel más bien parejo de Fl entre los casos, hay diferencias en sus componentes y entre sus perfiles: considerando una FI total de 0,68 con un máximo de 0,76 y un mínimo de 0,56, se puede justificar una intervención bastante estable. Al no contar con precedentes, sería poco aconsejable sugerir que el valor es suficiente o insuficiente para una intervención, pero, por definición, se asume que la FI aumenta con la implementación (Centruy et al., 2010). Dado el diseño de casos, se pueden detectar particularidades que hacen que la implementación muestre diferencias en la asimilación en cada colegio que sirven para una mejor gestión del proceso.

\section{Conclusiones}

Este primer estudio de fidelidad de implementación ( $\mathrm{Fl}$ ) de prácticas de liderazgo instruccional, como una cuantificación propuesta sobre la calidad del proceso, constituye uno de los aspectos más importantes para la investigación "marco" en que se desarrolla este estudio. Se busca una aproximación cuantitativa a la adopción de estas prácticas; por tanto, en un segundo momento podrá servir para medir progresos y constatar una mejora en la implementación, como señalan los antecedentes (Century et al., 2010).

Siguiendo las recomendaciones sobre los enfoques de implementación que se expresan en el marco teórico, quedó claro que la elección de un enfoque programado como la Fl era adecuada, por el grado de similitud (en estructura, tamaño y uso de artefactos curriculares y de gestión) que presentaban los seis casos (Fullan, 1983). Además, la metodología diseñada de intervención en terreno, uso de entrevistas, focus group abiertos y validación de los resultados con los equipos permitió sobrellevar las principales desventajas señaladas de no reconocer el fenómeno de forma completa (Altrichter, 2005).

En ese sentido, entrega criterios para una futura revisión de la FI dentro del proceso final de la intervención. Esto tiene alcances importantes para reconocer mejoras que se puedan producir en el proceso de intervención. Si se encuentran relaciones con otras variables de efecto, como motivación y rendimiento en estudiantes, el cruce con Fl será evidencia relevante de intervenciones de foco instruccional para el liderazgo. Como ya se revisó, y considerando una fidelidad de dos tercios $(0,68)$ de los elementos diseñados, se podrían considerar los cuadrantes de alta fidelidad 
de la matriz de Duvar (en O’Donnell, 2008). Esto favorece las decisiones de escalar la intervención tanto en el ámbito de las matemáticas de secundaria como en otras materias y niveles. A la vez, abre el campo para seguir investigando con este enfoque los proyectos que fortalecen el liderazgo instruccional u otro enfoque de influencia y mejora escolar.

Como una limitante a la validez interna, el instrumento aplicado solamente logró medir reportes, sin evidencias que sustentan lo declarado por los miembros ELI. En un futuro se recomendaría utilizar observaciones directas y analizar las herramientas y artefactos que se utilizan en las prácticas de liderazgo.

Otra limitante que abre una discusión consistió en que no todas las recomendaciones propias de la FI se cumplieron, sobre todo en cuanto al momento del diseño de la intervención, que en algunos aspectos fue posterior o simultáneo a esta medición. La investigación realizada asume como uno de sus focos el desarrollo ajustado a cada caso de las prácticas de influencia, según lo que hay o emerge de los equipos. Ello está contemplado también en las dimensiones bajo el concepto de "adaptación" o ajuste emergente (Century et al., 2010). Más allá, no siempre fue posible definir a priori la Fl del proceso, lo que hizo más relevante el diseño comparativo entre casos. Gracias a ello, se pudo diferenciar en qué escuelas y en qué aspectos se presentaban ventajas o mejores cumplimientos. Al no poder asegurar que la medición sea respecto una intención o diseño completo previo, la validez externa no es tan robusta como se desearía. Sin embargo, se espera contribuir al campo señalando que la FI en algunos casos puede fundamentarse mejor en la comparación de casos que en los diseños previos.

El hallazgo de la experiencia de mejora de la implementación en las prácticas y casos más débiles confirma lo anterior y refuerza el potencial de gestión del proyecto que este enfoque ofrece (Century et al., 2010). Surge la necesidad de contrastar esta idea con evaluaciones posteriores tanto de este proyecto como de cualquier otro.

Como se señalaba, el juicio de expertos, que usualmente proviene de los mismos ejecutores del proyecto, es crucial para tomar las decisiones de medición. Si estas decisiones están bien orientadas respecto a focos de medición y estándares esperables, la herramienta gana en utilidad. Sin embargo, y en concordancia con el párrafo anterior, una limitante en la FI es que el diseño de la evaluación no resuelve los problemas prácticos de implementación. En términos concretos, se podría medir con un modelo teóricamente muy sólido una intervención pobre. Dicha situación resulta tener un escaso valor práctico y presenta una limitante significativa para este tipo de investigaciones. Por otra parte, algunas de las críticas al modelo básico de fidelidad de implementación expuestas en los antecedentes del estudio son consistentes con esta experiencia, ya que durante una intervención de tres años en contextos reales es muy probable que sea necesario adaptar y ajustar criterios de aplicación durante el ejercicio de la misma (Reid et al., 1987).

En consecuencia, se refuerza la comparación de casos como estrategia de contraste y análisis de cómo un mismo plan se ajusta a la situación de intervención y cómo también la implementación opera reinterpretando los fundamentos teóricos de la intervención en el proceso de aplicación. Pareciera que en la literatura de la Fl este sustento teórico no se destacara suficientemente, como garante de que la intervención tenga relevancia. Por esta razón los focos más sólidos, como las prácticas de liderazgo instruccional (Robinson et al., 2008) y el foco en la mejora de la enseñanza matemática (Heck y Hallinger, 2009) fueron clave para mantener la relevancia y consistencia de esta investigación.

\section{Sobre los autores}

Germán Rodolfo Fromm-Rihm es licenciado en Psicología. Magíster y candidato a Doctor en Ciencias de la Educación de la Pontificia Universidad Católica de Chile. Becario de la Comisión Nacional de Investigación Científica y Tecnológica (Conicyt). Miembro del equipo de investigación del proyecto del Fondecyt No. 11100466 Influencia del liderazgo instruccional en motivación y logro académico en estudiantes secundarios.

Ingrid Marie Olbrich-Guzmán es profesora de Historia. Magíster en Dirección y Liderazgo Académico, Universidad del Desarrollo y Fundación Chile. Miembro del equipo de investigación del proyecto Fondecyt No. 11100466 Influencia del liderazgo instruccional en motivación y logro académico en estudiantes secundarios.

Paulo Luis Volante Beach es profesor Asociado de la Facultad de Educación de la Pontificia Universidad Católica de Chile. Doctor en Psicología Pontificia Universidad Católica de Chile. Investigador responsable del proyecto Fondecyt No. 11100466 Influencia del liderazgo instruccional en motivación y logro académico en estudiantes secundarios.

\section{Referencias}

Altrichter, H. (2005). Curriculum implementation-limiting and facilitating factors. In Context based learning of science (pp. 35-62). Waxmann: Munster.

Century, J., Rudnick, M. \& Freeman, C. (2010). A Framework for Measuring Fidelity of Implementation: A Foundation for Shared Language and Accumulation of Knowledge. American Journal of Evaluation, 31 (2), 199-218. 
Dumas, J., Lynch, A., Laughlin, J., Smith, E. \& Prinz, R. (2001). Promoting intervention fidelity: Conceptual issues, methods and preliminary results from the Early Alliance prevention trial. American Journal of Preventive Medicine, 20 (1), 38-47.

Fullan, M. (1983). Implementation und Evaluation von Curricula: USA und Kanada. In U. Hameyer, K. Frey y $\mathrm{H}$. Haft, Handbuch der Curriculumforschung (pp. 489-499). Beltz: Weinheim.

Hallinger, P. \& Heck, R. (2002). What Do You Call People With Visions? The Role of Vision, Mission and Goals in School Leadership and Improvement. In K. Leithwood \& P. Hallinger, Second International Handbook of Educational Leadership and Administration (pp. 9-40). Great Britain: Kluwer Academic.

Hallinger, P. \& Murphy, J. (1985). Assessing the Instructional Management Behavior the of principal. Elementary School Journal, 86 (2), 212-247.

Harris, A. (2009). Distributed Leadership: Different Perspectives. Netherlands: Springer.

Heck, R. H. \& Hallinger, P. (2009). Assessing the Contribution of Distributed Leadership to School Improvement and Growth in Math Achievement. American Educational Research Journal, 46 (3), 659-689. doi: 10.3102/0002831209340042

Hohmann, A. \& Shear, K. J. (2002). Community-based intervention research: Coping with the "noise" of real life in study design. American Journal of Psychiatry, 159, 201-207.

Hoy, W. S. (2002). Toward and Organizational Model of Achievement in High Schools: The Significance of Collective Efficacy. Educational Administration Quarterly, 38 (1), 77-93.

Leithwood, K. A. (1992). The move toward transformational leadership. Educational Leadership, 49 (5), 8-12.

Leithwood, K. A. \& Montgomery, D. J. (1980). Evaluating program implementation. Evaluation Review, 4, 193-214.

Leithwood, K. \& Riehl, C. (2005). What Do we Already Know About Educational Leadership? En W. Firestone \& C. Riehl, A New Agenda for Research in Educational Leadership. New York: Teachers College Press.

Leithwood, K., Day, C., Sammons, P., Harris, A. \& Hopkins, D. (2006). Successful School Leadership: What It Is and How It Influences Pupil Learning. Research Report RR800.
Marzano, R., Waters, T. \& Brian, M. (2005). School Leadership That Works: From Research to Results. USA: ASCD Association for Supervision and Curriculum Development.

Moncher, F. J. \& Prinz, R. J. (1991). Treatment fidelity in outcome studies. Clinical Psychology Review, 11, 247-266.

Mowbray, C., Holter, M., Teague, G. \& Bybee, D. (2003). Fidelity Criteria: Development, Measurement, and Validation. American Journal of Evaluation, 24, 315-340.

O’Donnell, C. (2008). Defining, Conceptualizing, and Measuring Fidelity of Implementation and Its Relationship to Outcomes in K12 Curriculum Intervention Research. Review of Educational Research, 78, 33-85.

Ontario Principal's Council (2009). The principal as Mathematics Leader. Ontario: Corwin Press.

Reid, K., Hopkins, D. \& Holly, P. (1987). Towards the effective school. Oxford: Blackwell.

Robinson, V., Lloyd, C. \& Rowe, K. (2008). The Impact of Leadership on Student Outcomes: An Analysis of the Differential Effects of Leadership Types. Educational Administration Quarterly , 44 (5), 635-674.

Schön, D. A. (1983). The Reflective Practitioner. London: Temple Smith.

Spillane, J. P., Halverson, R. \& Diamond, J. B. (2004). Towards a theory of leadership practice: a distributed perspective. Journal of Curriculum Studies, 36 (1), 3-34. doi: 10.1080/0022027032000106726

Spillane, J. P., Camburn, E. M. \& Pareja, A. S. (2007). Taking a distributed perspective to the school principal's workday. Leadership and Policy in Schools, 6 (1), 103-125.

Summerfelt, W. T. (2003). Program strength and fidelity to evaluation. Applied Developmental Science, 7, 55-61.

Teague, G. B., Drake, R. E. \& Ackerson, T. H. (1995). Evaluating use of continuous treatment teams for persons with mental illness and substance abuse. Psychiatric Services, 46 (7), 689-695.

Volante, P. (2010). Influencia instruccional de la dirección escolar en los logros académicos (Tesis Doctoral, Pontificia Universidad Católica de Chile).

Volante, P. (2011). Influencia del liderazgo instruccional en motivación y logro académico en estudiantes secundarios. Proyecto Fondecyt No. 11100466. Santiago: Pontificia Universidad Católica de Chile. 